\title{
The genetic diversity among strawberry breeding resources based on SSRs
}

\author{
Soohwan Lim¹, Jeongyeo Lee ${ }^{1}$, Hyun Joo Lee ${ }^{1}$, Kun-Hyang Park¹, Dae-Soo Kim¹, Sung Ran Min¹, Won Seok Jang², Tae II \\ $\mathrm{Kim}^{2}$, HyeRan Kim ${ }^{1,3 *}$
}

\author{
${ }^{1}$ Korea Research Institute of Bioscience and Biotechnology, \\ 125 Gwahangno, Yuseong-gu - Daejeon - 305-806 - \\ Republic of Korea. \\ ${ }^{2}$ Nonsan Strawberry Experiment Station/Chungcheong Nam- \\ Do Agricultural Research and Extension Services - Nonsan \\ - Chungnam - 320-862 - Republic of Korea. \\ 3University of Science and Technology/Systems and \\ Bioengineering, 217 Gajeong-ro - Daejeon - 34113 - \\ Republic of Korea. \\ *Corresponding author <kimhr@kribb.re.kr>
}

Edited by: Roberto Fritsche Neto

Received February 16, 2016

Accepted June 21, 2016

\begin{abstract}
Cultivated strawberry (Fragaria $\times$ ananassa Duch.) is a high value horticultural crop. In this study, the genetic diversity of 160 strawberry accessions was determined using five highly polymorphic simple sequence repeat (SSR) markers. Sixty different alleles were identified, with allele frequencies in the range of 0.006 to1. Similarity scores were in the range of 0.034 to 0.963 (average: 0.507 ). The accessions were categorized into five groups. Group 1 contained two diploid Fragaria vesca species and one unknown accession. Group 2 contained one accession ( $F . \times$ ananassa). Group 3 contained 20 F. $\times$ ananassa accessions and six unknown accessions. Group 4 contained 48 F. $\times$ ananassa accessions, one octaploid Fragaria chiloensis species, and six unknown accessions while Group 5 contained 69 F. $\times$ ananassa accessions and six unknown accessions. Accessions within a pedigree were frequently grouped together. $A$ total of 30 novel accessions were categorized alongside existing accessions. These results will allow breeders to develop strategies which incorporate more genetic diversity into new cultivars. Keywords: Fragaria $\times$ ananassa, simple sequence repeat, cultivar identification
\end{abstract}

\section{Introduction}

Cultivated strawberry (Fragaria $\times$ ananassa Duch.) is an octoploid $(2 \mathrm{n}=8 \mathrm{x}=56)$ generated by natural hybridization between two octoploid strawberry species, $F$. chiloensis and $F$. virginiana (Hancock et al., 2010). To date, 23 strawberry species have been reported, with various ploidy levels from diploid to decaploid (Nathewet et al., 2010; Rousseau-Gueutin et al., 2009).

Numerous strawberry breeding programs have been developed to generate new cultivars with improved taste and flavor or extended harvest period and shelf life (Capocasa et al., 2008; Faedi et al., 2000). Breeding success is associated with accessibility to breeding resources and genetic variations. Consequently, information regarding the genetic diversity and population structure of breeding resources is important for efficient development of new cultivars. Traditionally, the identification of strawberry cultivars was determined by the examination of differences in morphological or physiological characteristics such as leaf, flower, fruit parameters, and flowering habit (Dale, 1996; Nielsen and Lovell, 2000). However, such methods are unsuitable for cultivated strawberries as genetic and phenotypic variations between cultivars are minimal. As a consequence, DNA markers were developed to facilitate analysis of genetic diversity, linkage mapping, and identification of cultivars (Chambers et al., 2013; Congiu et al., 2000; Degani et al., 2001; Garcia et al., 2002; Govan et al., 2008; Isobe et al., 2013; Kunihisa et al., 2003; Tyrka et al., 2002). SSR markers for strawberry were first developed using primer pairs that amplified characterized regions such as expressed sequence tags (ESTs) or genomic libraries, in contrast to other primer pairs that amplified anonymous DNA fragments (Ashley et al., 2003; Bassil et al.,
2006; Gil-Ariza et al., 2006; James et al., 2003; Lewers et al., 2005; Monfort et al., 2006; Sargent et al., 2003). The completion of the genome sequence of diploid $F$. vesca (Shulaev et al., 2011) allowed robust SSRs to be developed and mapped on the Fragaria reference map (Rousseau-Gueutin et al., 2011; Sargent et al., 2011; Zorrilla-Fontanesi et al., 2011). In this study, we assessed the genetic diversity of 160 strawberry accessions used for breeding in Korea, including 34 newly collected accessions. The genotyping results will provide a molecular basis for future breeding programs and will facilitate the development of novel strawberry cultivars with increased genetic diversity.

\section{Materials and Methods}

\section{Plant materials and DNA extraction}

A total of 160 strawberry accessions (130 accessions from the Korea Rural Development Administration (RDA) Genebank Information Center, and 30 newly generated or collected accessions) were provided by the Nonsan Strawberry Experimental Station (Nonsan, Korea) (Table 1). Accessions were collected from Korea (26 accessions), Japan (46 accessions), the United States (45 accessions), Europe (13 accessions: four from the United Kingdom, three from the Netherlands, two each from Germany and Russia, and one each France and Spain), and other countries (nine accessions: three from each of Canada and Israel, two from China, and one from New Zealand). The countries of origin of 21 accessions were unknown. Genomic DNA was extracted from young leaves using a WizPrep Plant DNA Mini Kit (Wizbiosolutions) according to the manufacturer's protocol. The final DNA concentration was adjusted to $10 \mathrm{ng}$ per ul for use in a polymerase chain reaction (PCR). 
Table 1 - List of 160 strawberry accessions used in this study including accession name, origin, IT number, parentage, scientific name, and status.

\begin{tabular}{|c|c|c|c|c|c|c|}
\hline No. & Name & Origin & IT numbera & Parentage & Scientific name & Status \\
\hline 1 & Busan No.1101* & Korea, South & IT 232440 & Unknown & Fragaria $\times$ ananassa subsp. ananassa & Cultivar \\
\hline 2 & Chodong* & Korea, South & IT 232442 & Harunoka $\times$ Yachio & Fragaria $\times$ ananassa subsp. ananassa & Cultivar \\
\hline 3 & Daehak1* & Korea, South & IT 232439 & Unknown & Fragaria $\times$ ananassa subsp. ananassa & Cultivar \\
\hline 4 & Daeun* & Korea, South & IT 232453 & Akihime $\times$ Redpearl & Fragaria $\times$ ananassa subsp. ananassa & Cultivar \\
\hline 5 & Daewang $\mathrm{b}^{\mathrm{b}}$ * & Korea, South & IT 245989 & Maehyang $\times$ Wonkyo3111 & Fragaria $\times$ ananassa subsp. ananassa & Cultivar \\
\hline 6 & Daewang ${ }^{\mathrm{b},}$ * & Korea, South & IT 245989 & Maehyang $\times$ Wonkyo3111 & Fragaria $\times$ ananassa subsp. ananassa & Cultivar \\
\hline 7 & Dahong* & Korea, South & IT 232450 & Sachinoka $\times$ Maehyang & Fragaria $\times$ ananassa subsp. ananassa & Cultivar \\
\hline 8 & Danmi $^{*}$ & Korea, South & IT 245379 & Maehyang $\times$ Amaou & Fragaria $\times$ ananassa subsp. ananassa & Cultivar \\
\hline 9 & Geumhyang* & Korea, South & IT 232448 & Akihime $\times$ Tochiotome & Fragaria $\times$ ananassa subsp. ananassa & Cultivar \\
\hline 10 & Johong* & Korea, South & IT 232446 & Nyoho $\times$ Akihime & Fragaria $\times$ ananassa subsp. ananassa & Cultivar \\
\hline 11 & Maehyang* & Korea, South & IT 232445 & Tochinomine $\times$ Akihime & Fragaria $\times$ ananassa subsp. ananassa & Cultivar \\
\hline 12 & Manhyang* & Korea, South & IT 245381 & Nyoho $\times$ Akanekko & Fragaria $\times$ ananassa subsp. ananassa & Cultivar \\
\hline 13 & Mihong* & Korea, South & IT 232444 & Toyonoka $\times$ Reiko & Fragaria $\times$ ananassa subsp. ananassa & Cultivar \\
\hline 14 & NS970524* & Korea, South & IT 245343 & Unknown & Fragaria $\times$ ananassa subsp. ananassa & Line \\
\hline 15 & Okmae* & Korea, South & IT 245987 & Toyonoka $\times$ Maehyang & Fragaria $\times$ ananassa subsp. ananassa & Cultivar \\
\hline 16 & Seolhyang* & Korea, South & IT 232447 & Akihime $\times$ Redpearl & Fragaria $\times$ ananassa subsp. ananassa & Cultivar \\
\hline 17 & Suhong* & Korea, South & IT 232441 & Hokowase $\times$ Harunoka & Fragaria $\times$ ananassa subsp. ananassa & Cultivar \\
\hline 18 & Sulhong* & Korea, South & IT 232443 & Suhong $\times$ Toyonoka & Fragaria $\times$ ananassa subsp. ananassa & Cultivar \\
\hline 19 & Wildstrawberry $1^{*}$ & Korea, South & IT 245312 & Unknown & Fragaria $\times$ ananassa subsp. ananassa & Wild \\
\hline 20 & Wildstrawberry2* & Korea, South & IT 245335 & Unknown & Fragaria $\times$ ananassa subsp. ananassa & Wild \\
\hline 21 & Wonkyo3111* & Korea, South & IT 245867 & Open pollinated of Benihoppe & Fragaria $\times$ ananassa subsp. ananassa & Line \\
\hline 22 & Aiberry* & Japan & IT 232465 & Unknown & Fragaria $\times$ ananassa subsp. ananassa & Cultivar \\
\hline 23 & Aihime* $^{*}$ & Japan & IT 232463 & Unknown & Fragaria $\times$ ananassa subsp. ananassa & Cultivar \\
\hline 24 & Akanekko* & Japan & IT 232473 & Aiberry $\times$ Hokowase & Fragaria $\times$ ananassa subsp. ananassa & Cultivar \\
\hline 25 & Akasyanomitsuko & Japan & IT 245334 & Nyoho $\times$ Kunowase & Fragaria $\times$ ananassa subsp. ananassa & Cultivar \\
\hline 26 & Akihime & Japan & IT 245292 & Kunowase $\times$ Nyoho & Fragaria $\times$ ananassa subsp. ananassa & Cultivar \\
\hline 27 & Alps & Japan & IT 245363 & Unknown & Fragaria $\times$ ananassa subsp. ananassa & Cultivar \\
\hline 28 & Amaou & Japan & IT 232483 & Fukuoka S6 & Fragaria $\times$ ananassa subsp. ananassa & Cultivar \\
\hline 29 & Aska & Japan & IT 232470 & $\begin{array}{c}\text { Unknown } \\
\text { [Uzushio } \times\{(\text { Kurume Sokusei- } 3 \times \text { Hokowase }) \times\end{array}$ & Fragaria $\times$ ananassa subsp. ananassa & Cultivar \\
\hline 30 & Asuka Wave & Japan & IT 232480 & $\begin{array}{l}(\text { Donner } \times \text { Hokowase })\}] \times[(\text { Kurume Sokusei- } 3 \times \\
\text { Hokowase }) \times\{\text { Donner } \times(\text { Kobe- } 1 \times \text { Hokowase })\}]\end{array}$ & Fragaria $\times$ ananassa subsp. ananassa & Cultivar \\
\hline 31 & Bbiaberry & Japan & IT 245362 & Unknown & Fragaria $\times$ ananassa subsp. ananassa & Cultivar \\
\hline 32 & Benihoppe & Japan & IT 232482 & Akihime $\times$ Sachinoka & Fragaria $\times$ ananassa subsp. ananassa & Cultivar \\
\hline 33 & Chiduru & Japan & IT 232457 & [(Donner-s)-s $\times($ Harunoka-s)-s]-s $\times$ Harunoka-s & Fragaria $\times$ ananassa subsp. ananassa & Cultivar \\
\hline 34 & Terunoka & Japan & IT 232461 & Hokowase $\times$ Donner & Fragaria $\times$ ananassa subsp. ananassa & Cultivar \\
\hline 35 & Everberry & Japan & IT 245337 & Oishi-Sikinary $\times$ Haruyoi & Fragaria $\times$ ananassa subsp. ananassa & Cultivar \\
\hline 36 & Goryeong & Japan & IT 245328 & Takane Haikara $\times$ Donner & Fragaria $\times$ ananassa subsp. ananassa & Cultivar \\
\hline 37 & Guardian & Japan & IT 245289 & $\begin{array}{l}8[\text { Fairpeake } \times(\text { Aberdeen } \times \text { Redheart)] } \times \\
\text { Tennessee Beauty } \times \text { Surecrop }\end{array}$ & Fragaria $\times$ ananassa subsp. ananassa & Cultivar \\
\hline 38 & Harunoka & Japan & IT 232456 & Kurume103 $\times$ Donner & Fragaria $\times$ ananassa subsp. ananassa & Cultivar \\
\hline 39 & Haruyoi & Japan & IT 232460 & Hokowase $\times$ Harunoka & Fragaria $\times$ ananassa subsp. ananassa & Cultivar \\
\hline 40 & Hongbok & Japan & IT 245311 & Kohuku & Fragaria $\times$ ananassa subsp. ananassa & Cultivar \\
\hline 41 & Houkouwase $^{c}$ & Japan & IT 232455 & Kogyoku (Fairfax salf seedling) $\times$ Tahoe & Fragaria $\times$ ananassa subsp. ananassa & Cultivar \\
\hline 42 & Houkouwase ${ }^{c}$ & Japan & IT 232455 & Kogyoku (Fairfax salf seedling) $\times$ Tahoe & Fragaria $\times$ ananassa subsp. ananassa & Cultivar \\
\hline 43 & Jumbo & Japan & IT 232484 & Unknown & Fragaria $\times$ ananassa subsp. ananassa & Cultivar \\
\hline 44 & Jumbo Pure Berry & Japan & IT 232486 & Unknown & Fragaria $\times$ ananassa subsp. ananassa & Cultivar \\
\hline 45 & Kunowase & Japan & IT 245308 & Unknown & Fragaria $\times$ ananassa subsp. ananassa & Cultivar \\
\hline 46 & Kurume39 & Japan & IT 245327 & Unknown & Fragaria $\times$ ananassa subsp. ananassa & Line \\
\hline 47 & Kurume52 & Japan & IT 245332 & Unknown & Fragaria $\times$ ananassa subsp. ananassa & Line \\
\hline 48 & Myongbo & Japan & IT 245324 & Meiho derived & Fragaria $\times$ ananassa subsp. ananassa & Cultivar \\
\hline 49 & Nyoho & Japan & IT 232469 & Kei210 $\times$ Reiko & Fragaria $\times$ ananassa subsp. ananassa & Cultivar \\
\hline 50 & Pechikad $^{d}$ & Japan & IT 245346 & Unknown & Fragaria $\times$ ananassa subsp. ananassa & Cultivar \\
\hline 51 & Pechika $^{d}$ & Japan & IT 245346 & Unknown & Fragaria $\times$ ananassa subsp. ananassa & Cultivar \\
\hline 52 & Red Pearl & Japan & IT 232475 & Aiberry $\times$ Toyonoka & Fragaria $\times$ ananassa subsp. ananassa & Cultivar \\
\hline
\end{tabular}


Table 1 - Continuation.

\begin{tabular}{|c|c|c|c|c|c|c|}
\hline 53 & Regina & Japan & IT 245313 & Amerikanischer Samling II (Geneva) $\times$ Deutsch & Fragaria $\times$ ananassa subsp. ananassa & Cultivar \\
\hline 54 & Reiko & Japan & IT 232468 & Fukuba $\times$ Harunoka & Fragaria $\times$ ananassa subsp. ananassa & Cultivar \\
\hline 55 & Reiyu & Japan & IT 232464 & Unknown & Fragaria $\times$ ananassa subsp. ananassa & Cultivar \\
\hline 56 & Rockyhara & Japan & IT 245329 & Unknown & Fragaria $\times$ ananassa subsp. ananassa & Cultivar \\
\hline 57 & Sachinoka & Japan & IT 232476 & Toyonoka x Aiberry & Fragaria $\times$ ananassa subsp. ananassa & Cultivar \\
\hline 58 & Sagahonoka & Japan & IT 232458 & Unknown & Fragaria $\times$ ananassa subsp. ananassa & Cultivar \\
\hline 59 & Shinyurbong & Japan & IT 245336 & Unknown & Fragaria $\times$ ananassa subsp. ananassa & Cultivar \\
\hline 61 & Sungkang 17 & Japan & IT 245321 & Morioka17 & Fragaria $\times$ ananassa subsp. ananassa & Line \\
\hline 62 & Sungkang19 & Japan & IT 245322 & Morioka19 & Fragaria $\times$ ananassa subsp. ananassa & Line \\
\hline 63 & Syuko & Japan & IT 232477 & Shizutakara x Haruyoi & Fragaria $\times$ ananassa subsp. ananassa & Cultivar \\
\hline 64 & Tochinomine & Japan & IT 232479 & (Florida69-266 × Reiko) $\times$ Nyoho & Fragaria $\times$ ananassa subsp. ananassa & Cultivar \\
\hline 65 & Tochiotome & Japan & IT 232472 & Kurume $49 \times$ Tochinomine & Fragaria $\times$ ananassa subsp. ananassa & Cultivar \\
\hline 66 & Toyonoka & Japan & IT 232466 & Himiko $\times$ Harunoka & Fragaria $\times$ ananassa subsp. ananassa & Cultivar \\
\hline 67 & Wonyuk & Japan & IT 245325 & Himiko derived & Fragaria $\times$ ananassa subsp. ananassa & Cultivar \\
\hline 68 & Aiberry(ARS) & United States & IT 245365 & Unknown & Fragaria $\times$ ananassa subsp. ananassa & Cultivar \\
\hline 69 & Armore & United States & IT 232488 & Blakemore $\times$ Aroma & Fragaria $\times$ ananassa subsp. ananassa & Cultivar \\
\hline 70 & Berry Stare & United States & IT 232501 & Unknown & Fragaria $\times$ ananassa subsp. ananassa & Cultivar \\
\hline 71 & Berry Stare & United States & IT 232501 & Unknown & Fragaria $\times$ ananassa subsp. ananassa & Cultivar \\
\hline 72 & Blackmore & United States & IT 245298 & Missionary $\times$ Howard 17 & Fragaria $\times$ ananassa subsp. ananassa & Cultivar \\
\hline 73 & Camarosa & United States & IT 232510 & Douglas $\times$ Cal 85.218-605 & Fragaria $\times$ ananassa subsp. ananassa & Cultivar \\
\hline 74 & Cardinal & United States & IT 232487 & Earlibelle $\times$ ARK 5063 & Fragaria $\times$ ananassa subsp. ananassa & Cultivar \\
\hline 75 & Cascade & United States & IT 232503 & Shasta $\times$ Northwest & Fragaria $\times$ ananassa subsp. ananassa & Cultivar \\
\hline 76 & Catskill & United States & IT 232491 & Unknown & Fragaria $\times$ ananassa subsp. ananassa & Cultivar \\
\hline 77 & Columbia & United States & IT 232500 & WA $157 \times$ WA 175 & Fragaria $\times$ ananassa subsp. ananassa & Cultivar \\
\hline 78 & Comet & United States & IT 232499 & Earlibelle $\times$ ARK 5063 & Fragaria $\times$ ananassa subsp. ananassa & Cultivar \\
\hline 79 & Cyclone & United States & IT 232492 & Unknown & Fragaria $\times$ ananassa subsp. ananassa & Cultivar \\
\hline 80 & Dabreak & United States & IT 245299 & $\begin{array}{c}\text { liner } \times\{\text { Klonmore } \times[\text { L-117-1-45-3 } \times \text { (Suwan- } \\
\text { nee } \times \text { Konvoy) }]\}\end{array}$ & Fragaria $\times$ ananassa subsp. ananassa & Cultivar \\
\hline 81 & Diamante & United States & IT 245796 & Cal. 87112-6 × Cal. 88270-1 & Fragaria $\times$ ananassa subsp. ananassa & Cultivar \\
\hline 82 & Donner & United States & IT 245291 & CAL $222 \times$ CAL 145.52 & Fragaria $\times$ ananassa subsp. ananassa & Cultivar \\
\hline 83 & Earlibrite $^{f}$ & United States & IT 245382 & Rosalinda $\times$ FL 90-38 & Fragaria $\times$ ananassa subsp. ananassa & Cultivar \\
\hline 84 & Earlibrite $^{f}$ & United States & IT 245382 & Rosalinda $\times$ FL 90-38 & Fragaria $\times$ ananassa subsp. ananassa & Cultivar \\
\hline 85 & Erie Star & United States & IT 245294 & Unknown & Fragaria $\times$ ananassa subsp. ananassa & Cultivar \\
\hline 86 & Flamenco & United States & IT 245349 & Evita $\times$ EMR077 & Fragaria $\times$ ananassa subsp. ananassa & Cultivar \\
\hline 87 & Floridabelle & United States & IT 232502 & Sequioa $\times$ Earlibelle & Fragaria $\times$ ananassa subsp. ananassa & Cultivar \\
\hline 88 & Huxley & United States & IT 232504 & Unknown & Fragaria $\times$ ananassa subsp. ananassa & Cultivar \\
\hline 89 & Juspa & United States & IT 245300 & Unknown & Fragaria $\times$ ananassa subsp. ananassa & Cultivar \\
\hline 90 & Klondike & United States & IT 245301 & $\begin{array}{c}\text { Unknown } \\
\text { Blakemore } \times(\text { Marshall } \times \text { Fendalcinno }) \times \text { Nich }\end{array}$ & Fragaria $\times$ ananassa subsp. ananassa & Cultivar \\
\hline 91 & Lassen & United States & IT 232506 & $\begin{array}{c}\text { Ohmer } \times(\text { Royal Sovereign } \times \text { Howard 17) } \times(\text { Mar- } \\
\text { shall } \times \text { Fendalcinno })\end{array}$ & Fragaria $\times$ ananassa subsp. ananassa & Cultivar \\
\hline 92 & Linn & United States & IT 232505 & MDUS $3184 \times$ ORUS & Fragaria $\times$ ananassa subsp. ananassa & Cultivar \\
\hline 93 & Mingoi & United States & IT 245353 & Unknown & Fragaria $\times$ ananassa subsp. ananassa & Cultivar \\
\hline 94 & Missionary & United States & IT 245302 & Unknown & Fragaria $\times$ ananassa subsp. ananassa & Cultivar \\
\hline 95 & NewYork884 & United States & IT 245297 & Unknown & Fragaria $\times$ ananassa subsp. ananassa & Cultivar \\
\hline 96 & North West & United States & IT 245303 & Unknown & Fragaria $\times$ ananassa subsp. ananassa & Cultivar \\
\hline 97 & NY1406 & United States & IT 245320 & Unknown & Fragaria $\times$ ananassa subsp. ananassa & Line \\
\hline 98 & Pink Panda & United States & IT 232511 & Unknown & Fragaria $\times$ ananassa subsp. ananassa & \\
\hline 99 & Premier & United States & IT 232509 & Unknown & Fragaria $\times$ ananassa subsp. ananassa & Cultivar \\
\hline 100 & Redgauntlet & United States & IT 245304 & New Jersey $1051 \times$ Auchincruive Climax & Fragaria $\times$ ananassa subsp. ananassa & Cultivar \\
\hline 101 & Red Glow & United States & IT 232489 & Fairland $\times$ Tennessee Shipper & Fragaria $\times$ ananassa subsp. ananassa & Cultivar \\
\hline 102 & Red Rich & United States & IT 232497 & Unknown & Fragaria $\times$ ananassa subsp. ananassa & Cultivar \\
\hline 103 & Sari & United States & IT 245354 & Unknown & Fragaria $\times$ ananassa subsp. ananassa & Cultivar \\
\hline 104 & Shasta & United States & IT 232495 & Marshall x Howard 17 & Fragaria $\times$ ananassa subsp. ananassa & Cultivar \\
\hline 105 & Sweet Charlie & United States & IT 245345 & FL 80-456 × Pajaro & Fragaria $\times$ ananassa subsp. ananassa & Cultivar \\
\hline 106 & Dahoe & United States & IT 244919 & Unknown & Fragaria $\times$ ananassa subsp. ananassa & Cultivar \\
\hline
\end{tabular}


Lim et al.

Table 1 - Continuation.

\begin{tabular}{|c|c|c|c|c|c|c|}
\hline 107 & Victoria & United States I & IT 232508 & Unknown & Fragaria $\times$ ananassa subsp. ananassa & Cultivar \\
\hline 108 & Bolero & United Kingdom I & IT 245348 & LA0988 $\times$ Selva & Fragaria $\times$ ananassa subsp. ananassa & Cultivar \\
\hline 109 & Dyd Babi & United Kingdom I & IT 245318 & Unknown & Fragaria $\times$ ananassa subsp. ananassa & Cultivar \\
\hline 110 & Jonson's Early & United Kingdom I & IT 245319 & Unknown & Fragaria $\times$ ananassa subsp. ananassa & Cultivar \\
\hline 111 & Jursey Belle & United Kingdom I & IT 245296 & $\begin{array}{c}(\text { Lupton } \times \text { Aberdeen) } \times \text { Fairfax } \times(\text { Pathfinder } \times \\
\text { Fairfax) }\end{array}$ & Fragaria $\times$ ananassa subsp. ananassa & Cultivar \\
\hline 112 & Cavalier & Canada & IT 232515 & Valentine (Howard $17 \times$ Vanguard) $\times$ Sparkle & Fragaria $\times$ ananassa subsp. ananassa & Cultivar \\
\hline 113 & Guards Man & Canada & IT 232496 & Claribel $\times$ Sparkle & Fragaria $\times$ ananassa subsp. ananassa & Cultivar \\
\hline 114 & Micmac & Canada & IT 232516 & Tioga $\times$ Guardsman S1 & Fragaria $\times$ ananassa subsp. ananassa & Cultivar \\
\hline 115 & Nowangg & Israel & IT 245351 & Unknown & Fragaria $\times$ ananassa subsp. ananassa & Cultivar \\
\hline 116 & Nowangg & Israel & IT 245351 & Unknown & Fragaria $\times$ ananassa subsp. ananassa & Cultivar \\
\hline 117 & Tamar & Israel & IT 232517 & Osogrande $\times$ Dorit & Fragaria $\times$ ananassa subsp. ananassa & Cultivar \\
\hline 118 & Chunseong & China & IT 245356 & Unknown & Fragaria $\times$ ananassa subsp. ananassa & Cultivar \\
\hline 119 & Gilgyung53 & China & IT 245307 & Unknown & Fragaria $\times$ ananassa subsp. ananassa & Line \\
\hline 120 & Senga Gigana & Germany & IT 232513 & Unknown & Fragaria $\times$ ananassa subsp. ananassa & Cultivar \\
\hline 121 & Senga Sengana & Germany & IT 232512 & Markee $\times$ Sieger & Fragaria $\times$ ananassa subsp. ananassa & Cultivar \\
\hline 122 & Elsanta & Netherlands । & IT 245875 & Gorella $\times$ Holiday & Fragaria $\times$ ananassa subsp. ananassa & Cultivar \\
\hline 123 & Sarian & Netherlands I & IT 245364 & Unknown & Fragaria $\times$ ananassa subsp. ananassa & Cultivar \\
\hline 124 & Bukaj & Russia & IT 245180 & Unknown & Fragaria $\times$ ananassa subsp. ananassa & Cultivar \\
\hline 125 & Kama & Russia & IT 245357 & Sengasengana $\times$ Cavlier & Fragaria $\times$ ananassa subsp. ananassa & Cultivar \\
\hline 126 & Favette & France & IT 245373 & $\begin{array}{c}\text { (Souvenir des Halles } \times \text { Regina) } \times(\text { Pocahontas } \times \\
\text { Aliso) }\end{array}$ & Fragaria $\times$ ananassa subsp. ananassa & Cultivar \\
\hline 127 & Toteuklip & Spain & IT 245355 & Unknown & Fragaria $\times$ ananassa subsp. ananassa & Cultivar \\
\hline 128 & Chiloensis & & IT 245331 & Unknown & Fragaria chiloensis & Cultivar \\
\hline 129 & Fragaria Wild & & IT 245316 & Unknown & Fragaria $\times$ ananassa subsp. ananassa & Wild \\
\hline 130 & Jasan & & IT 232493 & Unknown & Fragaria $\times$ ananassa subsp. ananassa & Cultivar \\
\hline 131 & Crop Station & Korea, South & & Unknown & & \\
\hline 132 & Jirisan Wild & Korea, South & & Unknown & & \\
\hline 133 & Sakyejul & Korea, South & & Shikisetzu Unpulished & Fragaria $\times$ ananassa subsp. ananassa & \\
\hline 134 & Ssanta & Korea, South & & Maehyang $\times$ Seolhyang & Fragaria $\times$ ananassa subsp. ananassa & \\
\hline 135 & Sukhyang & Korea, South & & Seolhyang $\times$ Maehyang & Fragaria $\times$ ananassa subsp. ananassa & \\
\hline 136 & Elan & Netherlands & & Fern $\times$ Rapella & Fragaria $\times$ ananassa subsp. ananassa & \\
\hline 137 & New Zealand & New Zealand & & Unknown & & \\
\hline 138 & Caminoreal & United States & & Cal $89.2307 \times$ Cal 90.2533 & Fragaria $\times$ ananassa subsp. ananassa & \\
\hline 139 & Festival & United States & & Rosalinda $\times$ Osogrande & Fragaria $\times$ ananassa subsp. ananassa & \\
\hline 140 & Florida & United States & & Unknown & & \\
\hline 141 & Fragaria Minnesota & United States & & Unknown & & \\
\hline 142 & Ventana & United States & & Cal $93.170-606 \times$ Cal 92.35-601 & Fragaria $\times$ ananassa subsp. ananassa & \\
\hline 143 & Bious & & & Unknown & & \\
\hline 144 & EMC & & & Unknown & Fragaria vesca & \\
\hline 145 & Island Wild & & & Unknown & & \\
\hline 146 & Jepter & & & Unknown & & \\
\hline 147 & Naoami & & & Unknown & & \\
\hline 148 & Niigata & & & Unknown & & \\
\hline 149 & No0241 & & & Unknown & & \\
\hline 150 & No04113 & & & Unknown & & \\
\hline 151 & No06155 & & & Unknown & & \\
\hline 152 & No06172 & & & Unknown & & \\
\hline 153 & No06182 & & & Unknown & & \\
\hline 154 & No06183 & & & Unknown & & \\
\hline 155 & No06183 & & & Unknown & & \\
\hline 156 & No0636 & & & Unknown & & \\
\hline 157 & No0641 & & & Unknown & & \\
\hline 158 & No0643 & & & Unknown & & \\
\hline 159 & UC1 & & & Unknown & Fragaria vesca & \\
\hline 160 & Wild Strawberry3 & & & Unknown & & \\
\hline
\end{tabular}

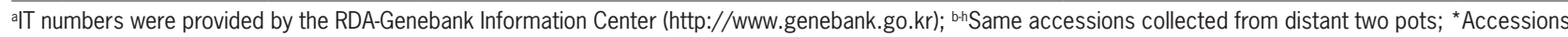
1-24 were used to select the SSR markers. 


\section{SSR analysis}

A subset of 24 strawberry accessions (accessions 1-24, Table 1) was used to select SSR markers that could discriminate effectively between the different accessions. Genotyping was performed using 16 SSR markers that were developed by three independent research groups (Chambers et al., 2013; Govan et al., 2008; Isobe et al., 2013). PCR products were analyzed using a MultiNA microchip electrophoresis system (MCE-202 MultiNA, Dongil Shimadzu). Peak sizes on electropherograms were automatically detected by MultiNA software (MultiNA Control Software and Data Analysis Software MultiNA Viewer, Dongil Shimadzu). Five SSR markers were selected from all the 160 strawberry accessions for analysis. Modified primer sequences for the markers are listed in Table 2. PCR reaction mixtures contained $1 \mathrm{ng}$ of template DNA, $1 \times$ PCR buffer, $0.25 \mathrm{mM}$ of dNTP mixture, $2.5 \mu \mathrm{M}$ of each primer, and 1 unit of i-Max II DNA polymerase (iNtRON) in a total volume of $20 \mu \mathrm{l}$. PCR conditions were as follows: initial denaturation at $94{ }^{\circ} \mathrm{C}$ for $5 \mathrm{~min}$, followed by 36 cycles at $94{ }^{\circ} \mathrm{C}$ for 30 $\mathrm{s}, 50{ }^{\circ} \mathrm{C}$ for $30 \mathrm{~s}$, and $72{ }^{\circ} \mathrm{C}$ for $30 \mathrm{~s}$, and then a final extension at $72{ }^{\circ} \mathrm{C}$ for $5 \mathrm{~min}$. PCR reactions were performed using a C1000 Touch Thermal Cycler (Bio Rad Laboratories).

\section{Genetic diversity analysis}

The genetic diversity of 160 strawberry accessions was analyzed using five selected SSR markers. First, SSR marker peaks were scored as 1 (presence of peak) or 0 (absence of peak) for each accession. Rare alleles and accession-specific alleles were applied in this study to distinguish strawberry accessions (Cho et al., 2007; Govan et al., 2008; Yoon et al., 2012). The binary data set for all strawberry accessions was then assembled in a single matrix and analyzed using the unweighted pair group method with an arithmetic mean (UPGMA, http:// genomes.urv.cat/UPGMA/) algorithm (using the Jaccard coefficient) to calculate genetic similarities and distances between the accessions. Output data in the Newick format were used with MEGA version 6 to produce a dendrogram (Tamura et al., 2013).

\section{Results and Discussion}

\section{Evaluation of diversity markers and genotyping of 160 strawberry accessions}

In this study, we strengthened strawberry breeding resources in Korea by analyzing 160 previously collected or newly generated strawberry germplasms. To determine the optimal markers for genotyping, 16 previously developed SSRs (nine from Govan et al. (2008), six from Chambers et al. (2013), and one from Isobe et al. (2013)| were used to test 24 of the 160 strawberry accessions (accession 1-24) (Table 1). Of the sixteen markers, eight yielded dim or difficult to interpret fingerprints, and three were insufficiently polymorphic (Figure 1). The remaining five markers, which were developed by Chambers et al. (2013), revealed clear fragments that were highly polymorphic between accessions (Figure 1). These five markers were chosen for genotyping from all 160 accessions. A total of 60 alleles (19 from FG7c/d, 13 from FG7a/b, 12 from FG1a/b, and 8 from each of FG2a/b and FG7e/f) were obtained from the five markers across all accessions (Table 2). All the markers were highly polymorphic: individual markers separated the 160 accessions into 17 (FG7e/f) to 54 (FG2a/b) allelic patterns. Allele sizes among the 160 accessions were in the range of $123 \mathrm{bp}$ (FG1a/b) to $599 \mathrm{bp}$ (FG7e/f) (Table 2), which differed slightly from those observed previously (Chambers et al., 2013). These differences may be attributable to the different strawberry accessions tested, slight modifications to primers (see Table 2), different PCR conditions, and different genotyping equipment.

Allele frequencies were in the $0.006-1$ range (Figure 2). Of the 60 alleles identified in the 160 accessions, 21 alleles (35\%) occurred with low frequency $(<0.1)$. These 'rare alleles' were detected by all five markers: one rare allele was identified using FG2a/b, three alleles from FG7e/f, five alleles from FG1a/b and FG7a/b, and seven alleles from FG7c/d (Table 2). One allele detected with FG7c/d (291 bp) was detected only in a single accession, accession 80 (from United States), which was suggestive of an accession-specific allele. Of the 160 strawberry accessions, $53 \%$ (84 accessions) possessed

Table 2 - List of five SSR primer pairs used for genotyping 160 strawberry accessions.

\begin{tabular}{|c|c|c|c|c|}
\hline $\mathrm{SSR}^{\mathrm{a}}$ & Primer sequences ${ }^{b}$ & No. of alleles scored & No. of rare alleles ${ }^{c}$ & Peak ranges observed (bp) \\
\hline $\mathrm{FGla} / \mathrm{b}$ & $\begin{array}{l}\text { Forward: TGGTTTGCCGGTAGCAAATAGCAGCA } \\
\text { Reverse : TGACACACACTCTCTCTGTCTGATCCCT }\end{array}$ & 12 & 5 & $123-167$ \\
\hline $\mathrm{FG} 2 \mathrm{a} / \mathrm{b}$ & $\begin{array}{l}\text { Forward : TGAACTGGTCCATCGGTGCTGAAA } \\
\text { Reverse : TGATCACACAATACGCATTACCAAGCCT }\end{array}$ & 8 & 1 & $298-362$ \\
\hline $\mathrm{FG7a} / \mathrm{b}$ & $\begin{array}{l}\text { Forward : GCAGTGCTACATCGACTCAGGTCCAA } \\
\text { Reverse : ACCAAGGAAGTGCCGAAGTGGGTTT }\end{array}$ & 13 & 5 & $153-240$ \\
\hline $\mathrm{FG7c} / \mathrm{d}$ & $\begin{array}{l}\text { Forward: AGGTGTCCAAAGAGGGTTGCTGTAGA } \\
\text { Reverse : TCCCTCTCCCAATAACCCTTTGCTTC }\end{array}$ & 19 & 7 & $236-396$ \\
\hline FG7e/f & $\begin{array}{l}\text { Forward : ACGGTGCCGAGATGCCTGATTACT } \\
\text { Reverse : GCTGATCTCCACTTCCTCTCCTATCACCA }\end{array}$ & 8 & 3 & $472-599$ \\
\hline
\end{tabular}




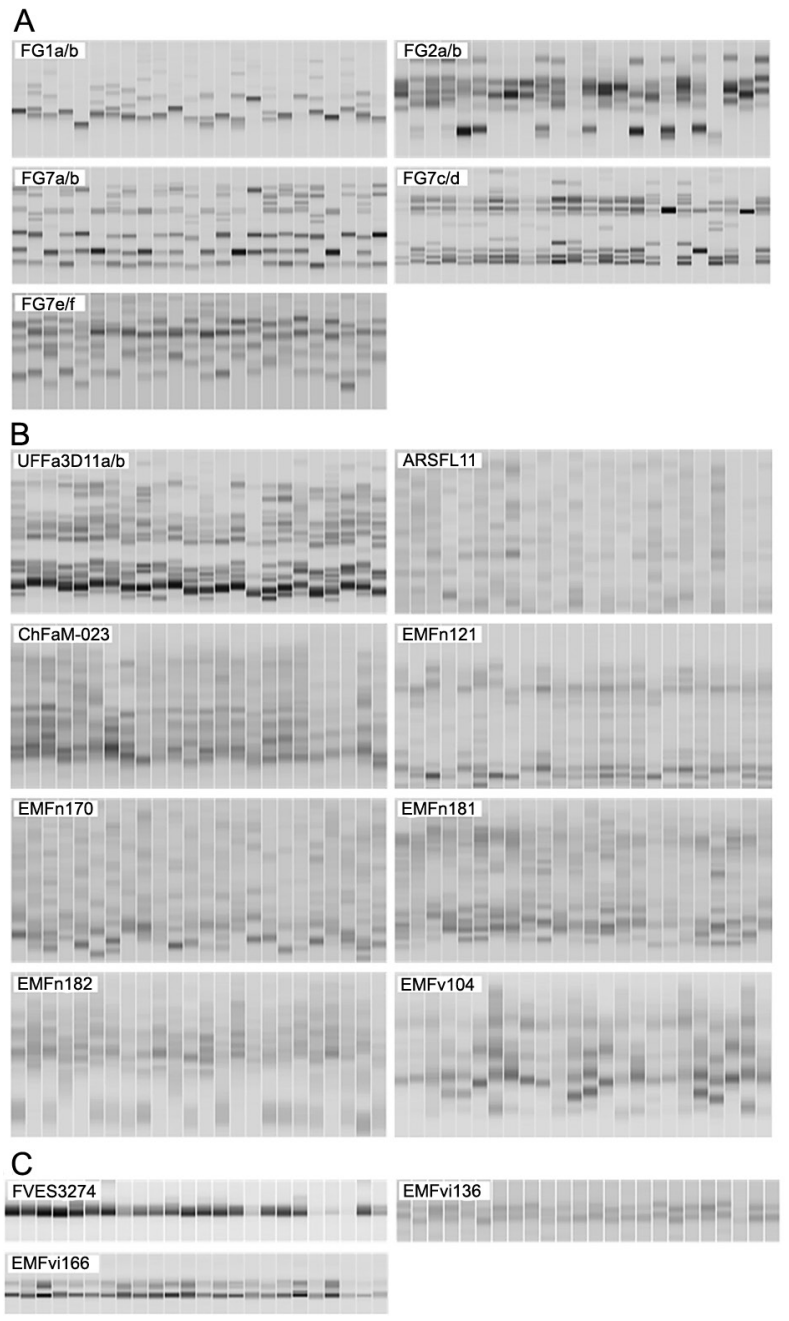

Figure 1 - Gel images of 24 strawberry accessions examined by 16 SSR markers. (A) Clear and highly polymorphic markers. (B) Dim or difficult to interpret markers. (C) Few polymorphic markers.

1-8 rare alleles, indicating the value of the rare alleles in discerning the different accessions. Of these 84 accessions, the largest number (32) was collected from the United States, followed by Japan (19), unknown origin (12), Korea (10), Europe (8), Canada (1), China (1), and New Zealand (1). One of the two Chinese accessions (accession 119) possessed five rare alleles, whereas the other Chinese accession (accession 118) showed no rare allele. This result indicated that the origin of the germplasm and the genetic relationship did not always correlated in strawberry resources.

\section{Genetic diversity and classification of strawberry accessions}

The genetic relationships between 160 strawberry accessions were analyzed using the 60 polymorphic alleles generated from the five SSR markers (Figure 3). Together, the five SSR markers distinguished 155 of the

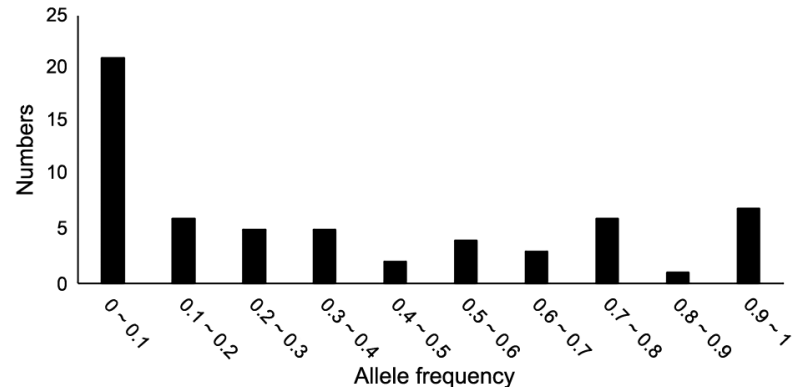

Figure 2 - A histogram of allele frequencies for 60 alleles in 160 strawberry accessions.

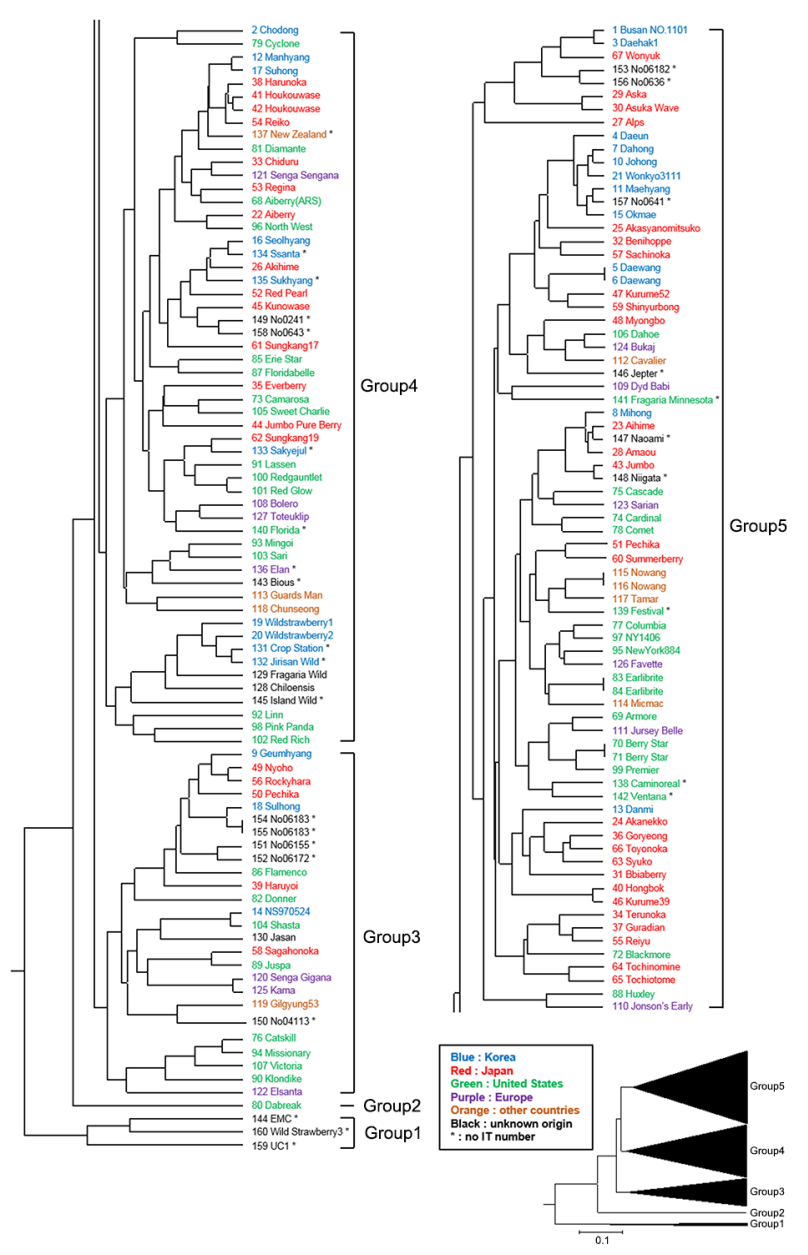

Figure 3 - Classification of 160 strawberry accessions based on their molecular diversity. Genetic similarities were calculated by UPGMA and the dendrogram was constructed using MEGA V.6.

accessions. The remaining accessions consisted of five pairs of duplicates (accession 83 (including its duplicate, accession 84), accession 115 (including its duplicate, accession 116), accession 70 (including its duplicate, accession 71), accession 5 (including its duplicate, accession 6), and accession 154 (including its duplicate, accession 
155)| that appeared to have the same allelic patterns for the five SSR markers (Figure 3). These results supported the effectiveness of our genotyping approach. However, two additional duplicates (accession 41 (including its duplicate, accession 42) and accession 50 (including its duplicate, accession 51)l, which were collected separately, appeared to be of different genotypes. The two accessions 41 and 42 were similar but not identical (Figure 3), and both belonged to group 4. One allele (302 bp from FG7c/d) was present in accession 41 but was absent in accession 42. The two accessions 50 and 51 were genetically distant and belonged to groups 3 and 5 , respectively (Figure 3). These two accessions differed by 10 alleles, suggesting that they were from different strawberry lines.

Excluding the five duplicate pairs, the genetic similarities of the strawberry accessions were in the range 0.034-0.963, with an average value of 0.507 . The highest similarity value (0.963) was between accession 148 (unknown origin) and accession 43 (from Japan), and between accession 125 (from Russia) and accession 120 (from Germany). The lowest similarity value (0.034) was between accession 159 (unknown origin) and accession 119 (from China).

The 160 strawberry accessions were divided into five groups according to genetic distance (Figure 3). Group 1 contained two diploid $F$. vesca species (accession 159 and accession 144) and one new accession, accession 160. These accessions were dissimilar to other accessions and were genetically distant showing the lower genetic similarities with other accessions 10.1 , $0.19,0.16$ for accession 159, accession 144, and accession 160 , respectively) compared to the average genetic similarities among the 160 accessions (0.509). These three accessions possessed relatively few SSR alleles 15 in accession 159, 11 in accession 144, and 9 in accession 160), compared to the average from all accessions (21.9). This was consistent with the simpler genetic structure found in diploid species such as $F$. vesca compared to octoploid species ( $F$. chiloensis and $F$. $\times$ ananassa). These data suggested that accession 160 belonged to $F$. vesca or another diploid species. Group 2 contained only one accession, accession 80, which was collected from the United States (Figure 3). One accession-specific allele (291 bp at FG7c/d) was detected in accession 80, alongside a further 16 SSR alleles.

Group 3 contained $20 F . \times$ ananassa accessions 18 from the United States, 5 from Japan, 3 each from Korea and Europe, and 1 from China) and six unknown origin accessions (Figure 3). In this group, several accessions were closely grouped in line with their geographical origins. For example, three accessions from the United States (accession 94, accession 107, and accession 90) and three from Japan (accession 49, accession 56, and accession 50 (including its duplicate, accession 51)) were closely grouped. Four of the six unknown origin accessions (accession 154 (including its duplicate, accession 155), accession 152, and accession 151) were also closely grouped, allowing for speculation that these may have originated in the same country. Of the 26 accessions in group 3, $16(62 \%)$ possessed at least one rare allele, which distinguishes the $F . \times$ ananassa accessions in this group from those in other groups.

The remaining 130 accessions were clustered into Groups 4 and 5, indicating minimal variability among these accessions within groups. Group 4 contained $48 \mathrm{~F}$. $\times$ ananassa accessions (17 from United States, 13 from Japan, 11 from Korea, 4 from Europe, and 1 each from Canada, China, and New Zealand), one octoploid species (F. chiloensis), and six unknown origin accessions (Figure 3). F. chiloensis, which is one of the parents of $F . \times$ ananassa cultivars, was located in a subcluster with three wild strawberry accessions (accession 19, accession 20, and accession 129) and three new accessions (accession 131, accession 132, and accession 145).

Group 5 contained $69 \mathrm{~F} . \times$ ananassa accessions $(27$ from Japan, 19 from the United States, 12 from Korea, 6 from Europe, 3 from Israel, and 2 from Canada) and six unknown origin accessions (Figure 3 and Table 1). Our five SSR markers confirmed the pedigree of some accessions such as accession 17 (the offspring of accession 41 and accession 42) and accession 38, and accession 54 (the grandparent of accession 12) (Figure 3 and Table 1). In addition. as shown in Figure 3 and Table 1, accession 134 was the offspring of accession 16, accession 26, and accession 52 were parents of accession 135, and accession 45 was one of the parents of accession 26. Certain accessions that shared at least one parent were also closely clustered. For example, accession 74 and accession 78 were both offspring of 'Earlibelle' and 'ARK5063'. Accession 139 and accessions 83 (including its duplicate, accession 84) shared the parent 'Rosalinda', and accession 139 and accession 117 shared the parent 'Osogrande' (Figure 3 and Table 1). By contrast, some accessions were in different groups despite sharing parents. For example, accession 39 (group 3) and accession 17 (group 4) were both offspring of accession 38 and 'Hokowase' (Figure 3 and Table 1). This result confirmed the previous observations of these accessions that clustered into the different group (Cho et al., 2007). Similarly, accession 16 (group 4) and accession 4 (group 5) were in different groups despite sharing parents (accession 26 and accession 52). This latter result was unexpected, as previous research had placed accession 16 and accession 4 in the same group (Hong et al., 2014) which might be due to the use of different batches of strawberry accessions or the number of accessions compared in two studies.

To date, most reports classifying Korean strawberry resources have examined only small numbers of accessions, such as those registered in the RDA-Genebank Information Center (http://www.genebank.go.kr). In this study, we presented an expanded dendrogram classifying 160 Korea strawberry breeding accessions, including 30 new, unregistered accessions. These new accessions were distributed throughout the dendrogram: three accessions in group 1, five in group 3, twelve in group 4, 
and ten in group 5. Further phenotypic classification of the new accessions, coupled with our marker-associated analysis, will enhance their value in breeding programs. Many of the accessions characterized in this study were generated by preexisting breeding programs, and consequently exhibited minimal genetic variation. The characterization of 160 strawberry accessions in the study will allow breeders to design new breeding strategies to incorporate more genetic diversity into new cultivars.

\section{Acknowledgments}

This work was supported by grants from the export promotion technology development research programs (315047-3) funded by the Ministry of Agriculture, Food and Rural Affairs, Republic of Korea, and a grant from the KRIBB Research Initiative Program.

\section{References}

Ashley, M.; Wilk, J.; Styan, S.; Craft, K.; Jones, K.; Feldheim, K.; Lewers, K.; Ashman, T. 2003. High variability and disomic segregation of microsatellites in the octoploid Fragaria virginiana Mill.(Rosaceae). Theoretical and Applied Genetics 107: 1201-1207.

Bassil, N.; Gunn, M.; Folta, K.; Lewers, K. 2006. Microsatellite markers for Fragaria from 'Strawberry Festival' expressed sequence tags. Molecular Ecology Notes 6: 473-476.

Capocasa, F.; Diamanti, J.; Tulipani, S.; Battino, M.; Mezzetti, B. 2008. Breeding strawberry (Fragaria $x$ ananassa Duch) to increase fruit nutritional quality. Biofactors 34: 67.

Chambers, A.; Carle, S.; Njuguna, W.; Chamala, S.; Bassil, N.; Whitaker, V.M.; Barbazuk, W. B.; Folta, K.M. 2013. A genomeenabled, high-throughput, and multiplexed fingerprinting platform for strawberry (Fragaria L.). Molecular Breeding 31: 615-629.

Cho, K.; Rho, I.; Cho, Y.; Park, P. 2007. Identification of Korean strawberry cultivars using DNA markers. Korean Journal of Breeding Science 40: 401-407.

Congiu, L.; Chicca, M.; Cella, R.; Rossi, R.; Bernacchia, G. 2000. The use of random amplified polymorphic DNA (RAPD) markers to identify strawberry varieties: a forensic application. Molecular Ecology 9: 229-232.

Dale, A. 1996. A key and vegetative descriptions of thirty-two common strawberry varieties grown in North America. Advanced Strawberry Research 15: 1-12.

Degani, C.; Rowland, L.J.; Saunders, J.A.; Hokanson, S.C.; Ogden, E.L.; Golan-Goldhirsh, A.; Galletta, G.J. 2001. A comparison of genetic relationship measures in strawberry (Fragaria $\times$ ananassa Duch.) based on AFLPs, RAPDs, and pedigree data. Euphytica 117: 1-12.

Faedi, W.; Mourgues, F.; Rosati, C. 2000. Strawberry breeding and varieties: situation and perspectives. Acta Horticulturae 567: 51-59.

Garcia, M.; Ontivero, M.; Diaz Ricci, J.; Castagnaro, A. 2002. Morphological traits and high resolution RAPD markers for the identification of the main strawberry varieties cultivated in Argentina. Plant Breeding 121: 76-80.
Gil-Ariza, D.; Amaya, I.; Botella, M.; Blanco, J.M.; Caballero, J.; López-Aranda, J.; Valpuesta, V.; Sánchez-Sevilla, J. 2006. EST-derived polymorphic microsatellites from cultivated strawberry (Fragaria $\times$ ananassa) are useful for diversity studies and varietal identification among Fragaria species. Molecular Ecology Notes 6: 1195-1197.

Govan, C.; Simpson, D.; Johnson, A.; Tobutt, K.; Sargent, D. 2008. A reliable multiplexed microsatellite set for genotyping Fragaria and its use in a survey of $60 F . \times$ ananassa cultivars. Molecular Breeding 22: 649-661.

Hancock, J.F.; Finn, C.E.; Luby, J.J.; Dale, A.; Callow, P.W.; Serçe, S. 2010. Reconstruction of the strawberry, Fragaria $\times$ ananassa, using genotypes of $F$. virginiana and F. chiloensis. HortScience 45: 1006-1013.

Hong, J.; Cho, K.; Kwon, Y. 2014. Construction of DNA profile data base of strawberry cultivars using microsatellite markers. Korean Journal of Horticultural Science 32: 853-863.

Isobe, S.N.; Hirakawa, H.; Sato, S.; Maeda, F.; Ishikawa, M.; Mori, T.; Yamamoto, Y.; Shirasawa, K.; Kimura, M.; Fukami, M. 2013. Construction of an integrated high density simple sequence repeat linkage map in cultivated strawberry (Fragaria $\times$ ananassa) and its applicability. DNA Research 20: 79-92.

James, C.; Wilson, F.; Hadonou, A.; Tobutt, K. 2003. Isolation and characterization of polymorphic microsatellites in diploid strawberry (Fragaria vesca L.) for mapping, diversity studies and clone identification. Molecular Ecology Notes 3: 171-173.

Kunihisa, M.; Fukino, N.; Matsumoto, S. 2003. Development of cleavage amplified polymorphic sequence (CAPS) markers for identification of strawberry cultivars. Euphytica 134: 209-215.

Lewers, K.; Styan, S.; Hokanson, S.; Bassil, N. 2005. Strawberry GenBank-derived and genomic simple sequence repeat (SSR) markers and their utility with strawberry, blackberry, and red and black raspberry. Journal of the American Society for Horticultural Science 130: 102-115.

Monfort, A.; Vilanova, S.; Davis, T.; Arús, P. 2006. A new set of polymorphic simple sequence repeat (SSR) markers from a wild strawberry (Fragaria vesca) are transferable to other diploid Fragaria species and to Fragaria $\times$ ananassa. Molecular Ecology Notes 6: 197-200.

Nathewet, P.; Hummer, K.E.; Yanagi, T.; Iwatsubo, Y.; Sone, K. 2010. Karyotype analysis in octoploid and decaploid wild strawberries in Fragaria (Rosaceae). Cytologia 75: 277-288.

Nielsen, J.; Lovell, P. 2000. Value of morphological characters for cultivar identification in strawberry (Fragaria $\times$ ananassa). New Zealand Journal of Crop and Horticultural Science 28: 89-96.

Rousseau-Gueutin, M.; Gaston, A.; Aïnouche, A.; Aïnouche, M.L.; Olbricht, K.; Staudt, G.; Richard, L.; Denoyes-Rothan, B. 2009. Tracking the evolutionary history of polyploidy in Fragaria L. (strawberry): new insights from phylogenetic analyses of low-copy nuclear genes. Molecular Phylogenetics and Evolution 51: 515-530.

Rousseau-Gueutin, M.; Richard, L.; Le Dantec, L.; Caron, H.; Denoyes-Rothan, B. 2011. Development, mapping and transferability of Fragaria EST-SSRs within the Rosodae supertribe. Plant Breeding 130: 248-255. 
Sargent, D.; Hadonou, A.; Simpson, D. 2003. Development and characterization of polymorphic microsatellite markers from Fragaria viridis, a wild diploid strawberry. Molecular Ecology Notes 3: 550-552.

Sargent, D.J.; Kuchta, P.; Girona, E.L.; Zhang, H.; Davis, T.M.; Celton, J.-M.; Marchese, A.; Korbin, M.; Folta, K.M.; Shulaev, V. 2011. Simple sequence repeat marker development and mapping targeted to previously unmapped regions of the strawberry genome sequence. The Plant Genome 4: 165-177.

Shulaev, V.; Sargent, D.J.; Crowhurst, R.N.; Mockler, T.C.; Folkerts, O.; Delcher, A.L.; Jaiswal, P.; Mockaitis, K.; Liston, A.; Mane, S.P. 2011. The genome of woodland strawberry (Fragaria vesca). Nature Genetics 43: 109-116.

Tamura, K.; Stecher, G.; Peterson, D.; Filipski, A.; Kumar, S. 2013. MEGA6: molecular evolutionary genetics analysis version 6.0. Molecular Biology and Evolution 30: 2725-2729.
Tyrka, M.; Dziadczyk, P.; Hortyński, J.A. 2002. Simplified AFLP procedure as a tool for identification of strawberry cultivars and advanced breeding lines. Euphytica 125: 273-280.

Yoon, M.-Y.; Moe, K.T.; Kim, D.-Y.; Rho, I.-R.; Kim, S.; Kim, K.-T.; Won, M.-K.; Chung, J.-W.; Park, Y.-J. 2012. Genetic diversity and population structure analysis of strawberry (Fragaria $\times$ ananassa Duch.) using SSR markers. Electronic Journal of Biotechnology 15: 6-6.

Zorrilla-Fontanesi, Y.; Cabeza, A.; Torres, A.M.; Botella, M.A.; Valpuesta, V.; Monfort, A.; Sánchez-Sevilla, J.F.; Amaya, I. 2011. Development and bin mapping of strawberry genicSSRs in diploid Fragaria and their transferability across the Rosoideae subfamily. Molecular Breeding 27: 137-156. 